\title{
Le droit de communiquer des salariés de la grand distribution dans l'espace de vente en vertu de l'article 7
}

\section{Risa L. Lieberwitz}

\section{(2) OpenEdition} Journals

Édition électronique

URL : https://journals.openedition.org/rdctss/1250

DOI : $10.4000 /$ rdctss. 1250

ISSN : 2262-9815

Éditeur

Centre de droit comparé du travail et de la sécurité sociale

Édition imprimée

Date de publication : 1 avril 2020

Pagination : 156-159

ISSN : $2117-4350$

\section{Référence électronique}

Risa L. Lieberwitz, «Le droit de communiquer des salariés de la grand distribution dans l'espace de vente en vertu de l'article 7 », Revue de droit comparé du travail et de la sécurité sociale [En ligne], 1 | 2020, mis en ligne le 01 novembre 2021, consulté le 11 novembre 2021. URL : http:// journals.openedition.org/rdctss/1250; DOI : https://doi.org/10.4000/rdctss.1250

\section{(c)}

Revue de droit comparé du travail et de la sécurité sociale est mise à disposition selon les termes de la Licence Creative Commons Attribution - Pas d'Utilisation Commerciale - Pas de Modification 4.0 International. 


\section{LE DROIT DE COMMUNIQUER DES SALARIÉS DE LA GRAND DISTRIBUTION DANS L'ESPACE DE VENTE EN VERTU DE L'ARTICLE 7}

Sur les cinq sièges que compte la Commission nationale des relations de travail (NLRB), trois seulement sont actuellement pourvus. Les trois membres actuels de la Commission sont des Conservateurs, nommés par le Président Trump et admis par un Sénat américain également conservateur. Les deux sièges vacants étaient jusqu'alors occupés par des commissaires plus libéraux, qui avaient été nommés par le Président Obama. L'Administration Trump n'a pas encore présenté de candidatures au Sénat pour pourvoir ces deux sièges après l'expiration du mandat des membres qui les occupaient.

La majorité de la Commission a maintenu sa tendance à restreindre les droits conférés aux salariés par la Loi nationale sur les relations de travail (NLRA) ${ }^{1}$, soit en annulant, soit en resserrant le champ d'application de ses jugements précédents. Elle a été très active, notamment dans la poursuite de sa série de décisions limitant le droit de communiquer librement sur les conditions de travail et la syndicalisation, reconnu aux salariés par l'article 7 de la NLRA. L'une des affaires a concerné la politique de l'entreprise Walmart sur le lieu de travail, qui accordait aux salariés le droit de porter « de petits logos ou signes n'attirant pas l'attention " tels que des boutons ou des broches de taille inférieure à celle de leur badge nominatif ${ }^{2}$. Certains salariés portaient des pin's affichant des messages de soutien au mouvement collectif «OUR Walmart » (Organization United for Respect at Walmart). La Commission a jugé légale la politique de Walmart restreignant le droit de communiquer des salariés dans un espace de vente de la grande distribution, en vertu de l'article 7. En revanche, elle a jugé que cette politique contrevenait à l'article 8(a)(1) de la NLRA lorsqu'elle était appliquée hors de l'espace de vente. S'il s'agit d'une victoire partielle pour les salariés, l'analyse de la Commission élargit le pouvoir de l'employeur de restreindre, sur le lieu de travail, les droits conférés aux salariés par l'article 7. La Commission a simplement mis en balance la justification commerciale invoquée par l'employeur pour sa politique, et l'impact de cette politique sur les droits des salariés. Cette approche dilue le critère juridique traditionnel qui exige que les employeurs prouvent l'existence de «circonstances particulières " pour pouvoir restreindre le droit de communiquer des salariés via le port de pin's ornés de messages ou d'autres insignes. Dans cette affaire, l'entreprise Walmart aurait dû être tenue de prouver la nécessité commerciale de réduire la taille des insignes à celle d'un petit badge nominatif. Au lieu de cela, la Commission a simplement accepté

129 U.S.C. $\S 151$ et seq.

2 Wal-Mart Stores, Inc., 368 NLRB n¹46 (2019). 
l'affirmation de Walmart selon laquelle le port d'un insigne plus apparent sur le lieu de vente aurait distrait les clients et compliqué l'identification des salariés de l'entreprise ${ }^{3}$.

La Commission a également fait preuve d'une grande déférence envers les employeurs dans deux autres décisions confirmant la validité des règles de confidentialité imposées sur le lieu de travail.

Dans l'affaire Apogee Retail LLC ${ }^{4}$, la Commission a jugé que l'employeur n'avait pas enfreint l'article $8(a)(1)$ de la NLRA en interdisant aux salariés de discuter des enquêtes en cours sur le lieu de travail, y compris les enquêtes relatives à des comportements illégaux ou contraires à l'éthique. La Commission a jugé que les règles de confidentialité des enquêtes étaient légales tant qu'elles stipulaient clairement ne s'appliquer que pour la durée de l'enquête. Elle a conclu que ces règles n'avaient qu'un faible impact sur le droit des salariés en vertu de l'article 7 de discuter de questions relatives au lieu de travail, et que les justifications de l'employeur en matière de confidentialité l'emportaient sur les intérêts des salariés. Néanmoins, la Commission a également décidé qu'elle évaluerait, au cas par cas, les règles de confidentialité qui ne se limitent pas aux enquêtes ouvertes. Dans de tels cas, elle mettra en balance les droits conférés aux salariés par l'article 7 et les justifications commerciales invoquées par l'employeur pour prolonger la durée de l'exigence de confidentialité. La Commissaire McFerran était en désaccord avec les trois membres majoritaires, faisant valoir que la Commission avait commis une erreur en annulant son propre précédent qui consistait à évaluer toutes les règles de confidentialité au travail au cas par cas, afin de déterminer si les justifications commerciales de l'employeur étaient légitimes ${ }^{5}$. McFerran s'inquiétait de l'effet dissuasif de ces règles sur la capacité des salariés à discuter sur le lieu de travail des questions faisant l'objet d'une enquête, et notamment sur le fait que les salariés faisant eux-mêmes l'objet d'une enquête puissent demander l'aide de collègues. McFerran a exprimé des inquiétudes similaires pour expliquer son désaccord avec une autre décision de la Commission qui jugeait que l'employeur n'enfreignait pas l'article 8(a) (1) en maintenant des règles qui obligeaient les salariés à garder confidentielles les listes de clients/ fournisseurs, et en interdisant aux salariés de fournir des informations sur les politiques de l'entreprise s'ils étaient approchés par les médias ${ }^{6}$. Comme l'a expliqué McFerran, la majorité de la Commission a ignoré le fait que l'employeur n'avait pas adapté plus étroitement ses règles de confidentialité pour protéger les droits conférés aux salariés par l'article 7, et leur permettre ainsi de partager des informations sur l'identité de leurs clients avec les syndicats des travailleurs, de contacter les clients de l'employeur pour obtenir leur soutien dans des conflits du travail ou de contacter les médias sur des questions liées au travail.

La Commission a poursuivi sa logique en accordant un poids excessif aux intérêts des employeurs en matière de propriété privée, au détriment des droits conférés aux salariés par l'article 7. Dans l'affaire Caesars Entertainment d/b/a/ Rio All-Suites Hotel and Casino7, la majorité de la Commission a jugé que les employeurs peuvent interdire aux salariés d'utiliser

3 La Commissaire McFerran, membre de la Commission nommée par Obama, faisait encore partie de la Commission lorsque l'affaire Walmart a été jugée. McFerran était en désaccord avec la décision de la majorité.

4368 NLRB n¹44 (2019).

5 Voir Banner Estrella Medical Center, 362 NLRB 1108 (2015).

6 LA Specialty Produce Company, 368 NLRB n 93 (2019).

7368 NLRB n¹43 (2019). 
les outils informatiques appartenant à l'employeur, y compris la messagerie électronique de l'entreprise, pour des communications ne concernant pas le travail. La majorité a renversé la décision de Purple Communications, Inc. ${ }^{8}$, qui avait jugé que les salariés autorisés à utiliser la messagerie électronique de l'entreprise avaient le droit, conformément à l'article 7, de l'utiliser à des fins non professionnelles en dehors des heures de travail. Les employeurs ne pouvaient limiter une telle utilisation que s'ils pouvaient prouver l'existence de « circonstances particulières » liées aux besoins de l'entreprise. Comme la Commissaire McFerran l'a noté dans sa dissidence sur l'affaire Caesars Entertainment, la façon dont la majorité a traité la messagerie électronique de l'entreprise ne tient pas compte de la réalité de l'utilisation quotidienne de la messagerie et impose des obstacles injustifiés aux salariés souhaitant exercer les droits conférés par l'article 7 pour communiquer entre eux sur des problèmes liés à leur environnement de travail.

Dans une autre décision récente, la Commission a rendu plus difficile pour les représentants des syndicats d'entrer dans les locaux appartenant à l'employeur. En vertu d'un précédent de longue date de la Cour suprême, les employeurs peuvent interdire aux représentants syndicaux d'accéder à des locaux leur appartenant, à condition d'appliquer leur règle de «non-sollicitation » de manière non discriminatoire ${ }^{9}$. Dans Kroger Mid-Atlantic ${ }^{10}$, la Commission a jugé avec une majorité de trois membres que l'entreprise une épicerie - avait légalement le droit d'interdire aux représentants syndicaux le parking du magasin où ils recueillaient des signatures de clients pour une pétition contre les pratiques de travail de l'employeur. La Commission a soutenu l'application par Kroger de sa règle de «non-sollicitation », bien que Kroger ait autorisé des organisations caritatives, telles que les Eclaireuses et l'Armée du Salut, à solliciter des dons et à vendre des articles sur ce même parking. En parvenant à cette conclusion, la Commission a infirmé l'une de ses décisions antérieures dans l'affaire Sandusky Mall Co. ${ }^{11}$, en vertu de laquelle l'action de Kroger envers les représentants syndicaux aurait été jugée discriminatoire. Dans sa décision sur l'affaire Kroger, la Commission a adopté une norme définissant la discrimination comme le fait que les employeurs traitent de façon inéquitable des activités de «nature similaire ». Selon cette définition, les représentants syndicaux qui se livraient à des activités syndicales ou à des manifestations ne participeraient pas à des activités similaires à celles à caractère caritatif, civique ou commercial des autres organisations autorisées à agir sur le parking de Kroger.

La NLRB a rendu plusieurs décisions s'appuyant sur les précédents de la Cour suprême des Etats-Unis confirmant le caractère exécutoire des décisions d'arbitrage des conflits liés à l'emploi sur les lieux de travail non syndiqués ${ }^{12}$. En vertu de ces décisions, les salariés renoncent à leur droit de demander réparation devant les tribunaux. En 2018, dans l'affaire Epic Systems Corp. v. Lewis ${ }^{13}$, la Cour suprême a jugé que les employeurs n'enfreignaient pas la NLRA en imposant des conventions d'arbitrage qui obligent les salariés à accepter que leurs différends liés à l'emploi soient résolus par le recours à un arbitre, renonçant ainsi au droit d'actions collectives. Dans une affaire ultérieure à Epic Systems, la NLRB a jugé que

8361 NLRB 1050 (2014).

9 Lechmere, Inc. v. NLRB, 502 U.S. 527 (1992); NLRB v. Babcock \& Wilcox, Inc., 351 U.S. 105 (1956).

10 Kroger Limited Partners/hip I Mid-Atlantic, 368 NLRB n64 (2019).

11329 NLRB 618 (1999).

12 Voir AT\&T Mobility LLC v. Concepcion, 563 U.S. 333 (2011); Circuit City Stores, Inc. v. Adams, 532 U.S. 105 (2001).

13138 S.Ct. 1612 (2018). 


\section{ÉTATS-UNIS}

les employeurs n'enfreignaient pas les droits conférés par l'article 7 s'ils imposaient des conventions d'arbitrage obligatoires forçant les salariés à arbitrer individuellement leurs différends liés à l'emploi, même si l'employeur élaborait ces dispositions contractuelles en réponse à une activité protégée des salariés ayant participé à une action collective sur les salaires et les horaires de travail14. La Commission a jugé, en outre, que les employeurs n'enfreignaient pas la NLRA en menaçant de licencier les salariés qui ne signeraient pas ces conventions d'arbitrage ${ }^{15}$. Cependant, elle a également jugé que les conventions d'arbitrage obligatoires ne peuvent pas légalement contraindre les salariés à renoncer à leurs droits de déposer des réclamations auprès de la NLRB ou d'autres instances administratives ${ }^{16}$. De plus, la Commission a jugé qu'un employeur enfreignait les droits conférés aux salariés par l'article 7 en imposant une convention d'arbitrage obligatoire qui reconnaissait aux salariés le droit de déposer des accusations de pratique déloyale de travail auprès de la NLRB, mais leur interdisait de recevoir un arriéré de paiement ou d'autres recours pécuniaires en cas de violation de la NLRA par l'employeur ${ }^{17}$.

14 Cordúa Restaurants, Inc., 368 NLRB n43 (2019).

15 ld. McFerran était en désaccord avec les deux jugements concernant Cordúa Restaurants.

16 Prime Healthcare Paradise Valley, LLC, 368 NLRB n¹0 (2019).

17 Kelly Services, Inc., 368 NLRB n¹30 (2019). 